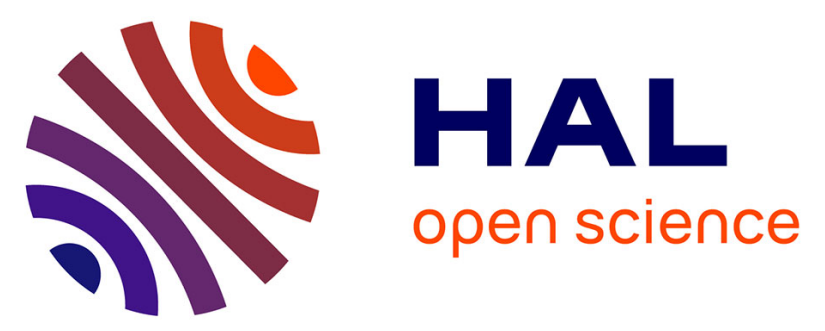

\title{
Redox and speciation micromapping using dispersive X-ray absorption spectroscopy: Application to iron in chlorite mineral of a metamorphic rock thin section
}

Manuel Munoz, Vincent de Andrade, Eric Lewin, Sakura Pascarelli, Jean Susini

\section{To cite this version:}

Manuel Munoz, Vincent de Andrade, Eric Lewin, Sakura Pascarelli, Jean Susini. Redox and speciation micromapping using dispersive X-ray absorption spectroscopy: Application to iron in chlorite mineral of a metamorphic rock thin section. Geochemistry, Geophysics, Geosystems, 2006, 7 (11), pp.Q11020. 10.1029/2006GC001381 . insu-00241847

\section{HAL Id: insu-00241847 https://hal-insu.archives-ouvertes.fr/insu-00241847}

Submitted on 11 Mar 2021

HAL is a multi-disciplinary open access archive for the deposit and dissemination of scientific research documents, whether they are published or not. The documents may come from teaching and research institutions in France or abroad, or from public or private research centers.
L'archive ouverte pluridisciplinaire $\mathbf{H A L}$, est destinée au dépôt et à la diffusion de documents scientifiques de niveau recherche, publiés ou non, émanant des établissements d'enseignement et de recherche français ou étrangers, des laboratoires publics ou privés. 


\title{
Redox and speciation micromapping using dispersive $\mathrm{X}$-ray absorption spectroscopy: Application to iron in chlorite mineral of a metamorphic rock thin section
}

\author{
Manuel Muñoz \\ Laboratoire de Géodynamique des Chaînes Alpines, Université Joseph Fourier, 1381 Rue de la Piscine, F-38041 \\ Grenoble,France(munoz@ujf-grenoble.fr) \\ European Synchrotron Radiation Facility, F-38043 Grenoble, France
}

Vincent De Andrade, Olivier Vidal, and Eric Lewin

Laboratoire de Géodynamique des Chaînes Alpines, Université Joseph Fourier, 1381 Rue de la Piscine, F-38041

Grenoble, France

\author{
Sakura Pascarelli and Jean Susini \\ European Synchrotron Radiation Facility, F-38043 Grenoble, France
}

[1] The first dispersive $\mu$-XANES mapping is presented here. The experiments have been conducted at the iron $K$-edge, on the "Dispersive-EXAFS" beamline of the European Synchrotron Radiation Facility (France). The mapping has been performed on a polished thin section of $30 \mu \mathrm{m}$ of a natural metamorphic rock including different types of minerals. Because of the high X-ray absorption due to the thickness of the glass sample holder $(\sim 1 \mathrm{~mm})$, the data have been collected in the fluorescence mode using the so-called "Turbo-XAFS" design. The effective spot size was approximately $10 \times$ 10 microns, and an area $390 \times 180$ microns in size was mapped. Improvements of the acquisition process allowed collection of each XANES spectrum in $1.5 \mathrm{~s}$ and with a step size of 5 microns so that 2808 spectra with full XANES information were collected. Then, automatic procedures for data reduction and mapping reconstruction were developed using Matlab ${ }^{\circledR}$. The results are maps of iron content, oxidation state, and speciation, with a $5 \mu \mathrm{m}$ spatial resolution after two-dimensional deconvolution. Subsequent analyses of the reconstructed images provide some quantitative calibrations.

Components: 5288 words, 4 figures.

Keywords: dispersive; XANES; mapping; redox; speciation; chlorite.

Index Terms: 3630 Mineralogy and Petrology: Experimental mineralogy and petrology; 3620 Mineralogy and Petrology: Mineral and crystal chemistry (1042); 3694 Mineralogy and Petrology: Instruments and techniques.

Received 6 June 2006; Revised 30 August 2006; Accepted 18 September 2006; Published 25 November 2006.

Muñoz, M., V. De Andrade, O. Vidal, E. Lewin, S. Pascarelli, and J. Susini (2006), Redox and speciation micromapping using dispersive X-ray absorption spectroscopy: Application to iron in chlorite mineral of a metamorphic rock thin section, Geochem. Geophys. Geosyst., 7, Q11020, doi:10.1029/2006GC001381. 


\section{Introduction}

[2] Mapping offers fundamental knowledge for heterogeneous samples as it integrates spot size information in a continuous two-dimensional space. Generally, chemical analyses using analytical methods like electron microprobe or X-ray fluorescence allow mapping of the concentration of elements. In situ information such as redox or speciation is generally accessible only locally, possibly at the micrometer scale depending on the size of the analytical probe. One of the most appropriate methods used to derive redox or speciation of a given atomic species in a wide variety of sample matrices is XANES (X-ray Absorption Near Edge Structure) spectroscopy. This method uses synchrotron X-ray sources and is element selective [e.g., Sayers et al., 1970; Lytle et al., 1975; Teo, 1986]. A typical acquisition time for the collection of a XANES spectrum is about 10 to $15 \mathrm{~min}$ in the most "standard" operation mode (i.e., "step-by-step" double-monochromator scanning process). Moreover, the data reduction is a critical step and automation is often complex. As a consequence, mapping of a sample using the "standard" microXANES technique involves measuring and treating several thousands of pixels (i.e., XANES spectra) and would require too much beam-time and data reduction time to be reasonably feasible. However, it is possible to collect X-ray absorption or fluorescence single-energy images from a monochromatic X-ray source. This technique allows visualizing contrasts for different energies relating to the oxidation state or the speciation of a given element (i.e., characteristic XANES features [see Ade et al., 1992; Sutton et al., 1995]). However, the recording of the full XANES information (i.e., high energy sampling rate, or "spectral resolution," with a high signal-to-noise ratio) would require the collection of a large number of images for the energy range (typically, a minimum of 500 for a standard XANES spectrum). Here again, the main limitation is the acquisition time, including all the difficulties related to the stability/reproducibility of the measurement (sample scanning, energy scanning, stability of the incident beam during a long period, etc.).

[3] Redox and speciation information is fundamental to characterize physicochemical mechanisms for a large variety of samples related to Physics, Chemistry, Biology or Earth Sciences. In this study, we have performed for the first time, "dispersive $\mu$-XANES mapping" of a com- plex geological sample in order to visualize the content, redox state and speciation variations of iron. The sample studied is a $30 \mu \mathrm{m}$ thin section of a metamorphic rock from the southwest of Japan (Sambagawa belt [see, e.g., Vidal et al., 2006]), which has chlorite, phengite and quartz as the main three minerals. The experiments were performed at the third generation synchrotron X-ray source of the ESRF (European Synchrotron Radiation Facility; France). More precisely, we used the microfocused "Dispersive-EXAFS" (Extended X-ray Absorption Fine Structure) beamline (ID24) in order to take advantage of the particularly high stability of the X-ray beam, and the very fast acquisition mode [Pascarelli et al., 2006]. Using this experimental setup, 2808 XANES spectra were collected at the iron $K$-edge $(7112 \mathrm{eV})$ within $104 \mathrm{~min}$ and with a $10 \times 10 \mu \mathrm{m}$ effective spot size FWHM (Full Width Half Maximum). Data were finally interpreted in terms of iron content, redox and speciation cartographies of $390 \times 180 \mu \mathrm{m}$ with a spatial resolution of $5 \mu \mathrm{m}$ after two-dimensional (2-D) deconvolution.

\section{Experimental Setup}

[4] Technical requirements to perform $\mu$-XANES mapping are (1) a microfocused X-ray probe, (2) a high stability of the incident beam, and (3) a very fast acquisition time. Thus a dispersive-EXAFS beamline such as ID24 at the ESRF is particularly well suited because the optical elements of the spectrometer are motionless during the experiments [e.g., Matsushita and Phizackerley, 1981; Buschert et al., 1988].

[5] In the present study, we used a silicon (311) bent monochromator (i.e., "energy dispersive polychromator") in the Bragg geometry. The energy range at the iron $K$-edge was optimized between 7100 and $7200 \mathrm{eV}$, in agreement with the settings of two insertion devices (undulators). A horizontal silicon mirror was positioned just before the sample in order to focus the spot vertically, and the sample chamber was evacuated to minimize the absorption of the air between the polychromator and the sample. Because of the high absorption of the glass sample holder $(1 \mathrm{~mm}$ thick) at the $\mathrm{Fe} K$-edge, data was collected in the fluorescence mode using the so-called "TurboXAS" (X-ray Absorption Spectroscopy) [Pascarelli et al., 1999]. A vertical slit located after the polychromator is used to scan continuously the horizontally dispersed X-ray beam (up to $40 \mathrm{~mm}$ length). 


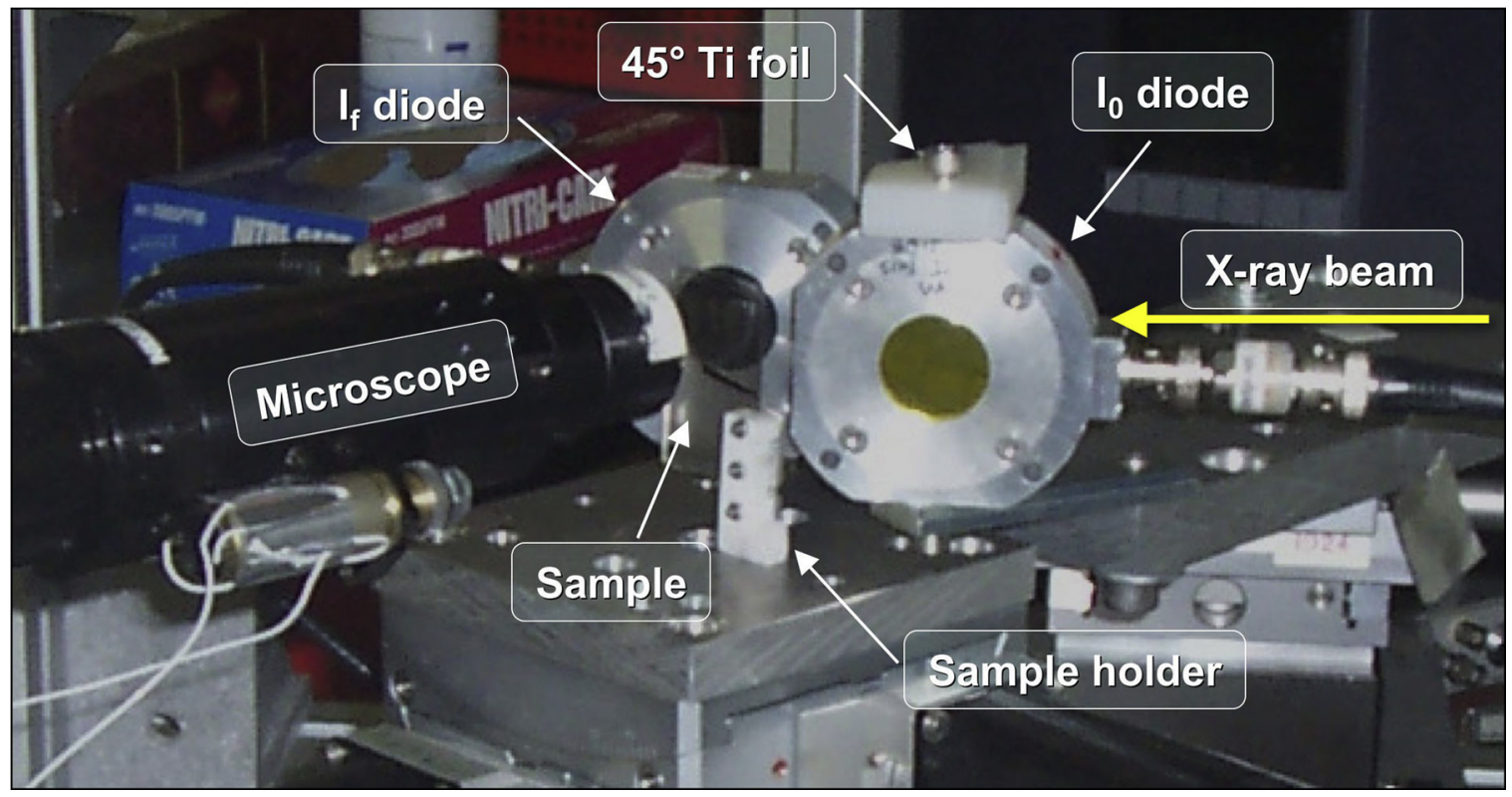

Figure 1. Experimental setup showing the direction of the X-ray beam, the geometry of the two diodes, and the microscope used to visualize the sample and localize the spot during data acquisition.

The incident beam is then monochromatic so that the fluorescence detection mode can be used. To reduce dead time during the acquisition, data were collected during both low-to-high and high-to-low energy scans. Using this experimental setup, a XANES spectrum can be collected in less than $1 \mathrm{sec}$. However, the acquisition time was here adjusted to $1.5 \mathrm{sec}$ to obtain sufficient statistics.

[6] Figure 1 presents the sample positioned at $45^{\circ}$ to the incident beam, the microscope at the rear of the sample, and the position of two diodes. During the acquisition, only the sample is mobile and motorized in its own plane so that (1) the angle of the incident beam is identical for every position and (2) there is no shift relative to the fixed focal spot of the X-ray beam. The microscope is used to accurately localize the beam on the sample's surface. The diode noted $\mathrm{I}_{0}$ is used to measure the intensity of the incident beam by way of the fluorescence of a titanium foil $6 \mu \mathrm{m}$ thick (disposed at $45^{\circ}$ of the diode). The diode noted $\mathrm{I}_{\mathrm{f}}$ is used to measure the fluorescence intensity, and is placed at $90^{\circ}$ of the incident beam. The incident beam passes between the two diodes spaced at approximately $10 \mathrm{~mm}$.

[7] The spot size at the focal distance was measured in the transmission mode, by scanning two gold wires of $5 \mu \mathrm{m}$ diameter. Gaussian fits reveal that the vertical beam size is about $10 \mu \mathrm{m}$ FWHM whereas the horizontal one is about $7 \mu \mathrm{m}$. However, because the sample is tilted by $45^{\circ}$ relatively to the incident beam, the effective irradiated horizontal length is around $10 \mu \mathrm{m}$. The effective spot size at the surface of the sample is thus about $10 \times 10 \mu \mathrm{m}$.

\section{Data Collection}

[8] The sample analyzed in this study is a metamorphic rock from Sambagawa, southwest of Japan. The sample is cut in a $30 \mu \mathrm{m}$ thin section and adhered to an iron-free glass holder $1 \mathrm{~mm}$ in thickness. Chlorite, phengite and quartz are the main minerals coexisting in this rock. Thus we expect large variations in iron content, redox state and speciation between the different species.

[9] On the basis of the optical image of the sample, a region of interest of $390 \mu \mathrm{m}$ by $180 \mu \mathrm{m}$ was defined on the sample, including the three different types of minerals. The mapping was performed by horizontal and vertical steps of $5 \mu \mathrm{m}$ so that 2808 XANES spectra were collected at the iron $K$-edge within $104 \mathrm{~min}$ (the collection of one spectrum requires $1.5 \mathrm{sec}$ acquisition $+0.7 \mathrm{sec}$ dead time due to mechanics and electronics). During the acquisition, the "raw data" are all stored in the same file as a succession of three columns: slit-encoder, $\mathrm{I}_{0}$ and $\mathrm{I}_{\mathrm{f}}$ values. A conver- 
sion between slit-encoder and energy values, based on a reference spectrum, is made during data reduction.

[10] Fe $K$-edge XANES spectra for metallic iron were collected in order to assure correct energy calibration and resolution. Moreover, different silicate standards like almandine and andradite were collected for the quantification of results. Each reference spectrum is the average of 10 acquisitions in order to improve the statistics required for subsequent pre-edge analysis.

\section{Data Reduction}

[11] The experimental setup described above yields a XANES spectrum that is first defined by "slit-encoder values" versus "absorbance values" (i.e., $\mathrm{I}_{\mathrm{f}} / \mathrm{I}_{0}$ ). Moreover, these values are measured every millisecond during the scan of the slit. Consequently, a XANES spectrum is over-sampled, and includes a few of thousands values. The first step of data processing consists of reducing the number of points per spectrum by averaging a given number of consecutive values. The spectra are then re-sampled at 520 points. This improves the signal-to-noise ratio and reduces the computing time for subsequent normalization and data analysis of the 2808 XANES spectra. The second step consists of the construction of two different 2-D matrixes. The first one includes the spectra collected from low-to-high energies (odd matrix), and the second one includes the spectra collected from high-to-low energies (peer matrix). The peer matrix is then "flipped vertically" in order to be comparable to the first one (i.e., all the data stored from low-to-high energies). The third step consists of interpolating XANES spectra for each matrix before calibrating on a similar energy range, between 7100 and $7200 \mathrm{eV}$. A singular 2-D matrix, including only one column of energy values, is then reconstructed alternating "odd" and "peer" spectra from the two different matrixes. The 2808 raw spectra resulting from this step are presented in Figure 2a.

[12] The normalization of the spectra is commonly performed in two phases. First, the background before the edge is modeled from 7100 to $7110 \mathrm{eV}$ using a linear function. The function is then subtracted to compensate the slope of the raw spectrum and to fix the pre-edge part to zero. Second, the XANES spectra are fitted using an arctangent function with fixed width $(2.2 \mathrm{eV})$ and variable position and height. This is done to normalize the edge-jumps to the arbitrary value of 1 . Moreover, the position and height values are stored in two different 2-D matrixes to reconstruct the images based on these two criteria. Also, a 3-D matrix is constructed including the two dimensions of the image and, as third dimension, the absorbance values of each XANES spectra. The 3-D matrix is used to visualize in 2-D the "absorbance contrast" at a given energy. Each resulting image is deconvoluted in order to improve the spatial resolution by a factor 2; the beam size being two times greater, horizontally and vertically, than the sampling step.

[13] Note that because of the high heterogeneity of iron content in the sample, the automation for the normalization procedure could not be done using the same initial parameters for all the spectra. Particularly, for the spectra with an extremely low signal-to-noise ratio (i.e., very low iron content), the pre-edge fit was constrained in order to limit an eventual excessive slope. Moreover, the energy range used to fit the arctangent function was limited to exclude possible distorted parts at the extremities of the spectra (i.e., compromise between the quality of normalization and the possible occurrence of aberrant normalization). The width of the arctangents was also fixed to $2.2 \mathrm{eV}$ to improve the normalization process. These additional constraints only affected less than $0.5 \%$ of the spectra that needed an individual normalization. The series of the 2808 normalized spectra is presented in Figure $2 b$ (distortions observed before the edge for scan numbers between 2000 and 3000 result from the surface rendering effect and occur from less than 10 highly noisy/ distorted spectra, with extremely low edge-jump of around $5.10^{-3}$ ).

\section{Results}

[14] Figure 3a presents a polarized optical image that includes the region mapped. Chlorite is the greenish to brownish mineral in the middle of the picture, quartz is the white mineral located close to the top, and phengite is located in between chlorite and quartz as well as at the bottom of the picture (i.e., the elongated and fibrous mineral). Note that quartz is relatively fractured and contains several interstitial minerals. Also, a quartz crystal, approximately 25 by $50 \mu \mathrm{m}$, is included in the chlorite. In parallel, Figure $3 \mathrm{e}$ provides the same image recorded in the crossed-polarizer mode. Here again, the different crystalline phases are clearly visible. Even so, this mode is particularly useful to 

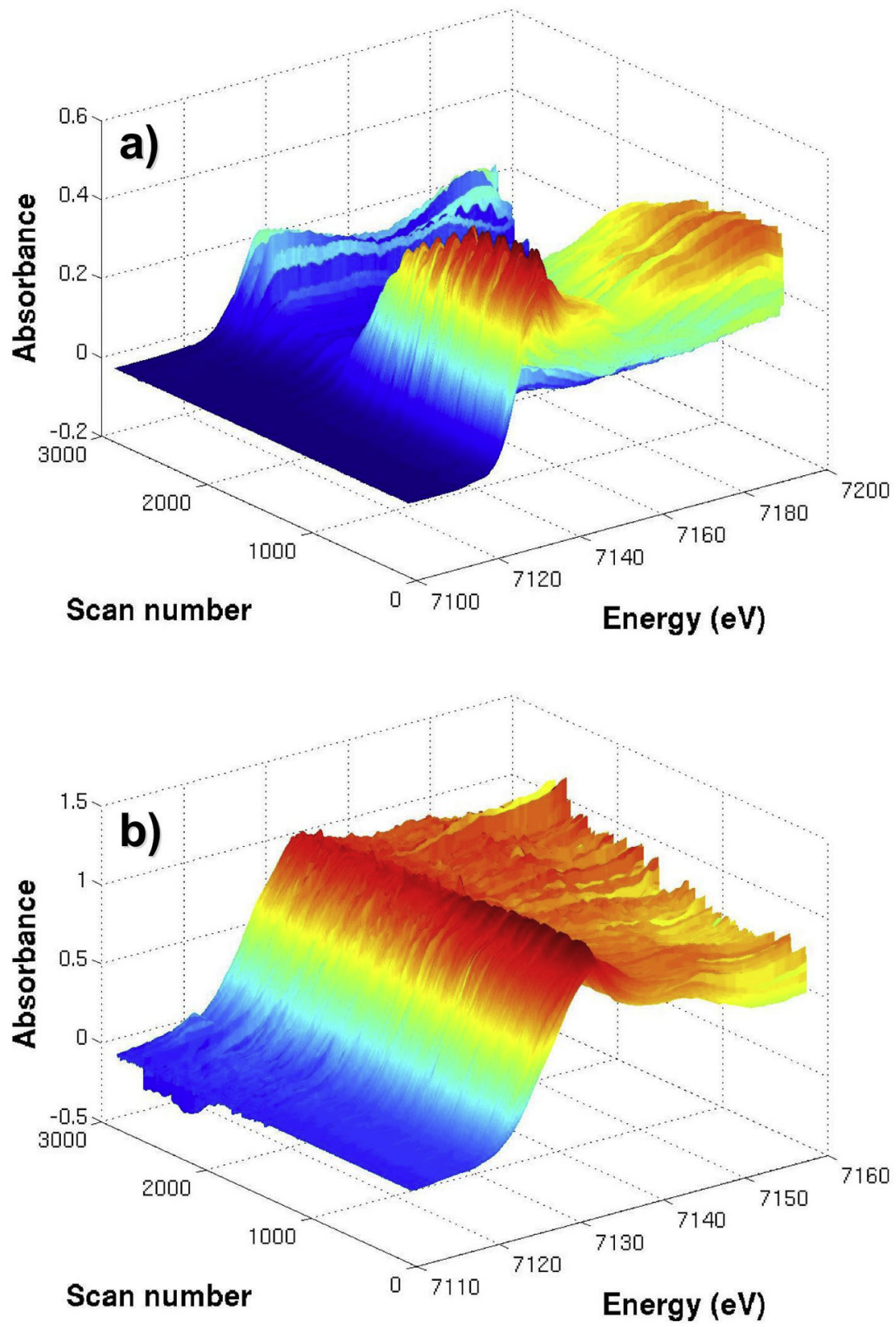

Figure 2. XANES spectra collected from the bottom to the top of the mapped region, alternatively from left to right and from right to left: (a) raw spectra and (b) normalized spectra.

qualitatively characterize changes in the orientation of crystals of a given mineralogical phase. Particularly, the chlorite phase highlights two main orientations of the crystals. A first one appears mostly in blue, and corresponds to the greenish part of the chlorite in Figure 3a. A second one appears essentially in green and orange, and corresponds to the most brownish regions of the chlorite in Figure 3a. One can thus deduce that chlorite crystals present here two main orienta- tions that appear directly correlated to changes in color on the "simply"-polarized optical image. Note that birefringence variations in chlorite are not necessarily due to different crystal orientations, but can also occur from differences in mineralogical composition and/or the brownish coloration of the mineral.

[15] The edge-jump values provided by the arctangent fits can be directly correlated to the total iron content of the sample [Teo, 1986; Muñoz et al., 

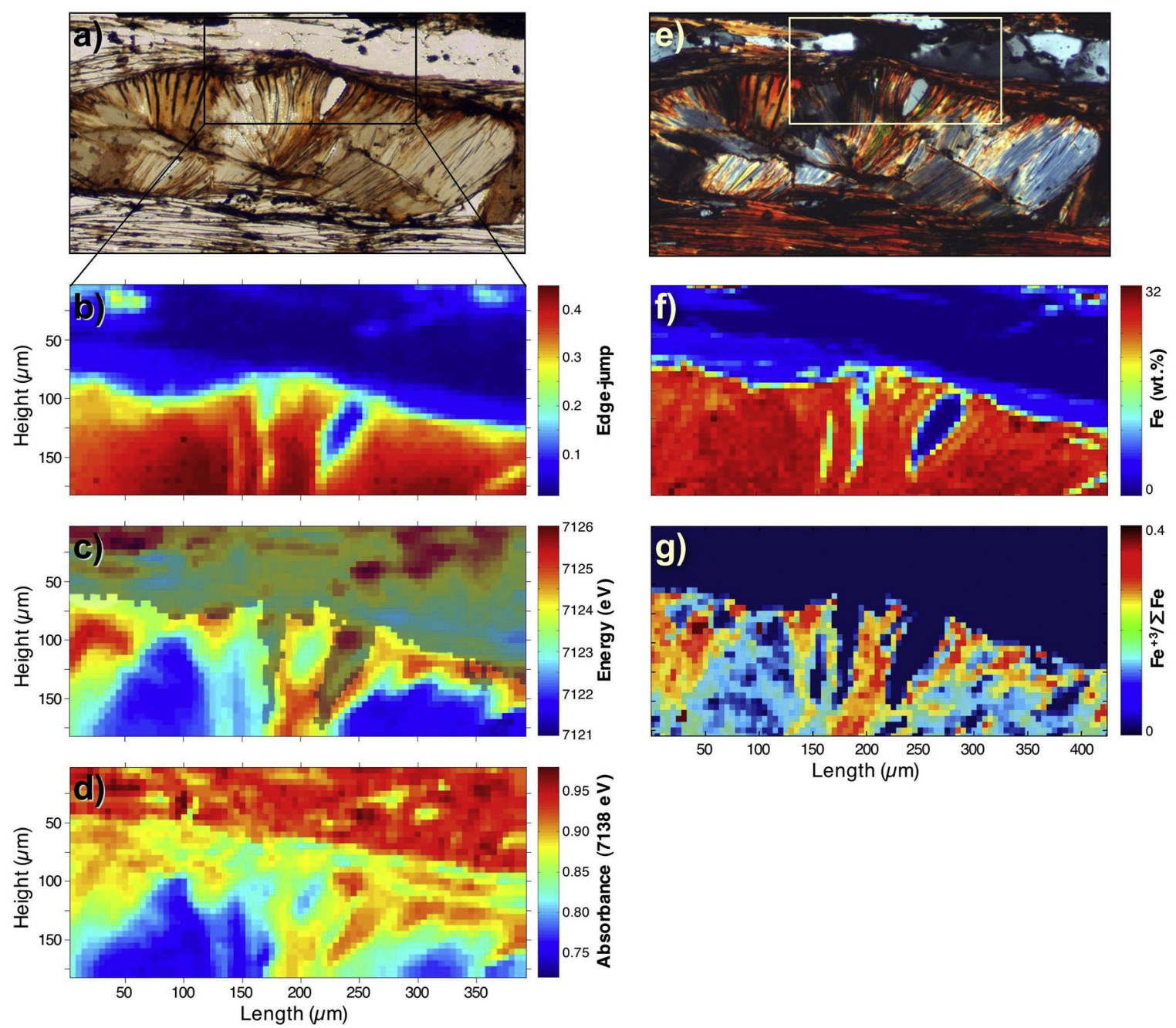

Figure 3. Rock sample mapping: (a) polarized optical image (chlorite in the middle, quartz at the top, and phengite between chlorite and quartz and at the bottom); (b) iron content (edge-jump); (c) iron redox (edge-position); (d) iron speciation (absorbance contrast at $7137 \mathrm{eV}$ ); (e) crossed-polarizer optical image; (f) quantitative iron content map (from electron microprobe analyses); and ( $\mathrm{g}$ ) quantitative iron redox map (from thermodynamics).

2005]. In the present case, XANES spectra are measured in the fluorescence mode so that selfabsorption for the different types of minerals possibly affects nonlinearly the fluorescence yield [e.g., Pfalzer et al., 1999]. Consequently, to assess the measurements and calibrate the XANES "edge-jump" information, we performed electron microprobe maps (see De Andrade et al. [2006] for details of data collection and quantification). Figure $3 \mathrm{~b}$ provides an iron content mapping based on the 2-D matrix containing the edge-jump values, whereas Figure $3 f$ shows a quantitative iron content map collected on the electron microprobe. The two images show very good correlation and the main difference results from the different size of the probes for the two techniques (i.e., a few microns for the electron microprobe versus $\sim 10 \mu \mathrm{m}$ for the X-ray beam). Results show a relatively high and homogeneous iron content in chlorite, with a maximum of $32 \mathrm{wt} . \%$. Also, the iron content in phengite is around 6 wt. $\%$ whereas quartz crystals show less than 5000 ppm of iron, most likely related to small inclusions. Such high variations of iron content clearly highlight the three different crystalline phases present in the region of interest.

[16] Similarly, a map can be reconstructed on the basis of the position of the inflection point of the arctangents assimilated to the energy of the absorption edge (Figure 3c). Indeed, this criterion can be directly correlated to the oxidation state of iron; the Fermi level being shifted of a few electron-volts with 

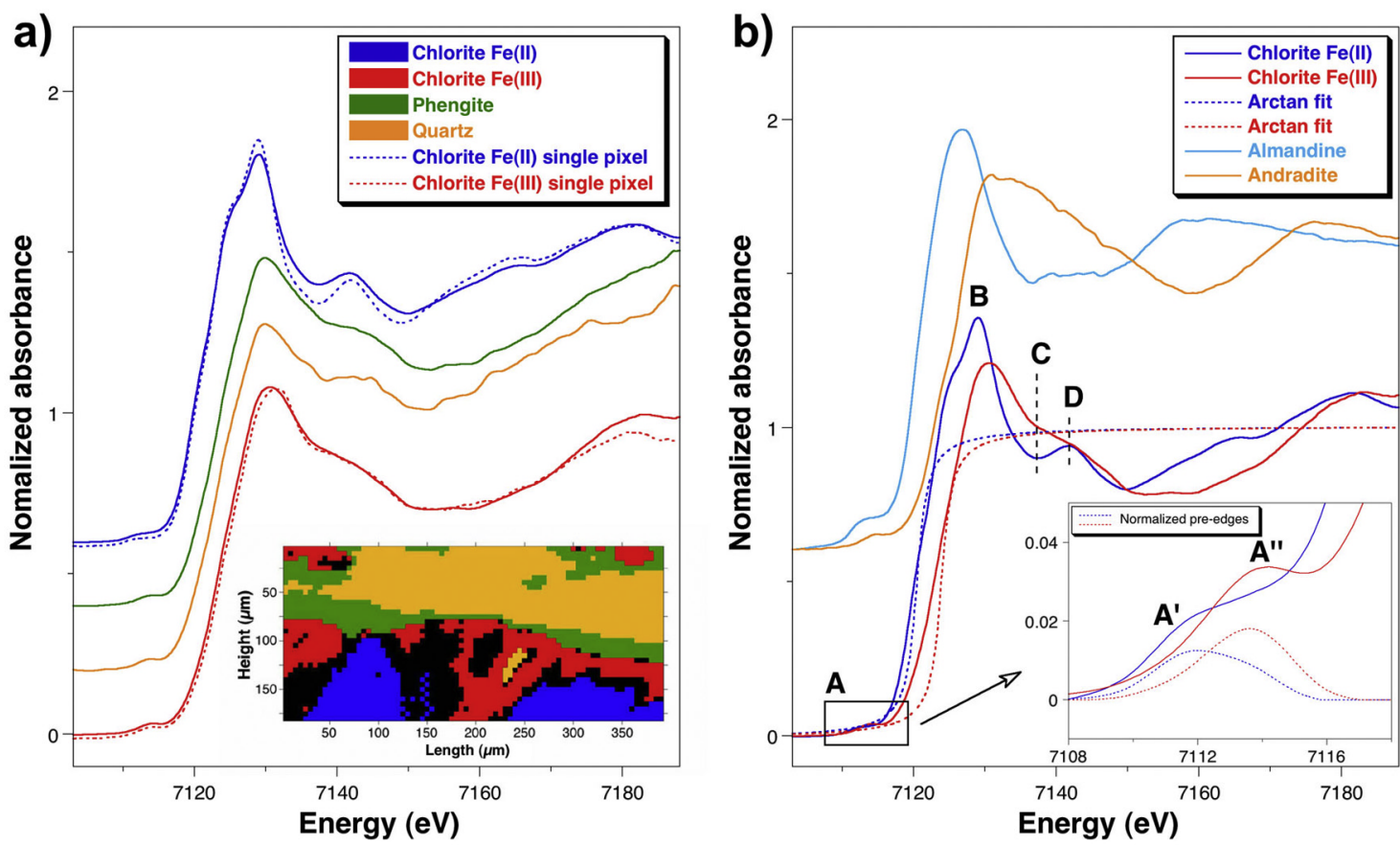

Figure 4. (a) Averaged and "single-pixel" XANES spectra based on the different masks of the maps. Blue, chlorite-Fe(II); green, phengite; orange, quartz; and red, chlorite-Fe(III). (b) XANES spectra for chlorite-Fe(II) and chlorite-Fe(III) with the arctangent fits (dashed lines) and a zoom on the pre-edges showing also their normalization (dashed lines). XANES spectra of almandine and andradite are shown to illustrate of the $K$-edge shift of reference compounds.

increased oxidation of cations [e.g., Ankudinov et al., 1998]. The mapping reveals a relatively high iron redox contrast in the sample, with a significant shift of the absorption edge (around $5 \mathrm{eV}$, from 7121 to $7126 \mathrm{eV}$ ). More particularly, the mapping qualitatively highlights a high heterogeneity within the chlorite: the center of the mineral being mostly reduced, whereas the borders, in contact with the phengite and the quartz inclusion being mostly oxidized. Such variations most likely occur from redox changes, though in the case of iron, a $1 s-4 s$ electronic transition possibly contributes to the absorption edge [Shulman et al., 1976; Berry et al., 2003]. Moreover, chlorite crystals corresponding to reduced and oxidized regions seem to present different orientations as suggested by the crossed-polarizer optical image (Figure 3e), and the linear polarization of the X-ray beam potentially affects the different features of the spectra, specifically when anisotropic crystals are studied [Manceau et al., 1988; Dyar et al., 2002a]. An accurate quantification of iron redox variations based on the edge position is thus difficult. Even so, though the post-edge and edge regions of the spectra may be significantly affected by the linear polarization of the X-ray beam, the influence on the pre-edge region does not appear to be significant [Dyar et al., 2002a]. Consequently, our quantification is based on pre-edge analysis. For the typical XANES spectra of the reduced and oxidized regions of the chlorite (see subsequent explanations related to Figure 4 for the selection of masks and the extraction of spectra from the images), a pre-edge analysis (i.e., $1 s-3 d$ electronic transitions; data reduction based on the method proposed by Wilke et al. [2001]) was performed in order to calibrate Figure 3c. The results provide pre-edge centroid positions of $7112.16 \mathrm{eV}$ and $7112.69 \mathrm{eV}$ for the more reduced and oxidized regions, respectively. According to Wilke et al. [2001], such values correspond to a variation of the $\mathrm{Fe}^{3+} / \sum \mathrm{Fe}$ ratio from 0.03 to 0.34 that can be associated, in the present case, to variations of the edge-position from 7121 to $7125 \mathrm{eV}$. In order to validate the technique and to estimate the error on pre-edge quantification related to the potential problems mentioned above, we provide a modeled image of iron oxidation state corresponding to the same region of interest (see Figure $3 \mathrm{~g}$ ). This image was obtained by coupling elemental maps 
collected on the electron microprobe and thermodynamic calculations (see Vidal et al. [2006] for details in a complementary study of the same sample). The maps of Figures $3 \mathrm{c}$ and $3 \mathrm{~g}$, obtained from fully independent methods, show an excellent correlation (note that the dark blue region of Figure $3 \mathrm{~g}$ is reported as a transparent blue mask in Figure $3 \mathrm{c}$ in order to highlight the chlorite phase). Moreover, the $\mathrm{Fe}^{3+} / \sum \mathrm{Fe}$ ratio provided by the theoretical approach ranges from 0 to 0.4 though it ranges from 0.03 and 0.34 for the present study. The error related to the XANES technique for the $\mathrm{Fe}^{3+} / \sum \mathrm{Fe}$ ratio is thus estimated to be less than 0.1 .

[17] A speciation map is finally presented in Figure 3d. This map is based on the variation of the absorbance at a given energy for the normalized XANES spectra. The energy was chosen to correspond to a characteristic feature highlighting the different atomic environment of iron; in the present case, $7137 \mathrm{eV}$ (see feature $\mathrm{C}$ in Figure 4b). Indeed, as shown subsequently, this energy presents a maximum in the variation of the absorbance depending on the iron speciation. The map shows a distinct difference between quartz (i.e., red region) and the other minerals whereas there is no clear distinction between chlorite and phengite. Moreover, the variations observed for the chlorite correlate with the ones observed on the redox mapping (i.e., Figure $3 \mathrm{c}$ ). Such information clearly suggests that divalent iron and trivalent iron present different XANES spectral signatures, and thus are located in two different types of crystallographic environments [e.g., Wilke et al., 2001; Dyar et al., 2002b]. However, some slight but significant differences can be observed between these two images, particularly in the region located downward, in the right part: the blue field appears slightly smaller in Figure 3d. This information suggests that though the $\mathrm{Fe}^{2+}$ is essentially located in a specific environment (different from the one of $\mathrm{Fe}^{3+}$ ), part of the $\mathrm{Fe}^{2+}$ can reside in the $\mathrm{Fe}^{3+}$ site in the structure. On the other hand, trivalent iron seems to be quasi-exclusively located in only one type of crystallographic environment.

[18] A next step in the interpretation of results consists of establishing different masks based on the maps presented in Figure 3. It is then possible to separate the regions corresponding to the different minerals and/or the different oxidation states of iron. The image inset in Figure 4a shows five different masks defined from the mapping. The green and orange masks have been determined on the basis of the variations in iron content (Figure 3b), and correspond to the regions of phengite and quartz, respectively. The blue and red masks have been determined on the basis of the variations of iron redox (Figure 3c), and correspond to the location of $\mathrm{Fe}^{2+}$ and $\mathrm{Fe}^{3+}$, respectively. The black mask corresponds to the region presenting a mix between $\mathrm{Fe}^{2+}$ and $\mathrm{Fe}^{3+}$, and is not used for subsequent analysis. It is then possible to reconstruct the XANES spectra corresponding to each region by averaging the spectra included in each pixel of the masks. This step of data analysis allows reconstructing "end-members" XANES spectra for the sample by increasing significantly their signal-to-noise ratio compared to a "singlepixel" XANES spectrum, which is required for quantitative pre-edge analyses. Thus four XANES spectra associated to the different masks (except the black one) are presented in Figure 4a: from the bottom to the top, "Fe $\mathrm{Fe}^{3+}$-chlorite," quartz, phengite and " $\mathrm{Fe}^{2+}$-chlorite." Note that for $\mathrm{Fe}^{3+}$, , and $\mathrm{Fe}^{2+}$-chlorite, single-pixel spectra are shown in dashed lines. The comparison with the averaged spectra shows a very good correlation although there is a slight decrease in the spectral resolution due to the slight redox/speciation heterogeneities within the red and blue masks. The spectra for $\mathrm{Fe}^{3+}$-chlorite, quartz, phengite are similar in terms of position of the absorption edge and spectral signature above the edge; the spectrum related to quartz however, is noisy because of the particularly low iron content. The two different types of spectra for chlorite reveal some major differences. The XANES spectra as well as their respective arctangent fits are shown in Figure 4b. The arctangent fits highlight the energy shift between the absorption edges, also illustrated by XANES spectra of almandine (ferrous iron standard) and andradite (ferric iron standard). The signal-to-noise ratio allows the analysis of the $\mathrm{Fe}-K$ pre-edge of the spectra (feature A; zoom inset in Figure 4b) so that, as previously detailed, the iron redox map could be quantified according to standard compounds. Also, the integrated intensity of the pre-edge revealed that both $\mathrm{Fe}^{2+}$ and $\mathrm{Fe}^{3+}$ are located in octahedral environments (see normalized pre-edges; respectively, feature $\mathrm{A}^{\prime}$ and $\mathrm{A}^{\prime \prime}$ in dashed lines in Figure $4 \mathrm{~b}$ ).

[19] From a crystallographic point of view, the qualitative analysis of the edge (feature B) and the XANES part of the spectra (mostly features $\mathrm{C}$ and D) qualitatively informs about the atomic 
environment of iron. Notably, the spectrum corresponding to the $\mathrm{Fe}^{2+}$ presents some clear characteristics of an octahedral environment with significant $\mathrm{Fe}-\mathrm{Fe}$ second-neighbor multiplescattering contributions (see Farges et al. [2001] for similarities with nickel). On the other hand, the spectrum corresponding to the $\mathrm{Fe}^{3+}$ appears much "smoother," suggesting less multiple-scattering interactions between the photoelectron and the structure, and possibly a lower atomic density in the second and third atomic shell around the trivalent iron. Though more specific ab initio XANES calculations would be required for a more robust site assignment of iron in chlorite, this interpretation suggests that trivalent iron would be preferentially located in the octahedral interfoliar layer of the chlorite, which is in good agreement with a thermodynamic approach of the crystallography of chlorites [Vidal et al., 2005].

\section{Summary}

[20] This work presents a new and original method to characterize in 2-D the redox and the speciation of iron by performing a "full information" XANES mapping. This type of analysis became possible thanks to third generation synchrotron $\mathrm{X}$-ray beamlines, such as the dispersive-EXAFS beamline ID24 at the ESRF, able to provide a high photon flux within a microfocused spot and allowing extremely fast XANES acquisition, both in the transmission and in the fluorescence modes. First, the XANES mapping provides both, iron redox and speciation maps. A second step of analysis allows discriminating the different regions of interest of the sample, and averaging the XANES spectra corresponding to these regions. Thus the improvement of the signal-to-noise ratio makes possible a quantitative study useful for calibrating the different types of reconstructed images.

[21] The potential of this new tool is illustrated by studying a complex geological material (metapellite rock including quartz, phengite and chlorite) in which the distribution of iron is highly heterogeneous. The results show a high contrast in the oxidation state of iron, particularly within the chlorite mineral. Moreover, the analysis of the iron speciation in the chlorite reveals that divalent iron and trivalent iron are most likely located in two different crystallographic environments in the structure.

[22] Such an analytical tool can potentially find wide application in Earth Sciences, but also in
Physics, Chemistry or Biology; the present results being here essential for modeling the thermodynamics "out of equilibrium" of metamorphic rocks [Vidal et al., 2006]. A further step in the development of the dispersive X-ray absorption spectroscopy micromapping technique will consist of collecting data up to the EXAFS region in order to provide some quantitative speciation maps (i.e., interatomic distances, coordination numbers, Debye-Waller factors, etc.). These developments will beget the adaptation of automatic procedures for EXAFS data reduction; including standard normalization and background subtraction, as well as analyses based on the Continuous Cauchy Wavelet Transform (CCWT) [see Muñoz et al., 2003].

\section{Acknowledgments}

[23] We thank all the staff of the ESRF (Grenoble, France) that made these developments possible, in particular, S. Pasternak and F. Perrin for the excellent technical support and M. C. Dominguez for the development of specific acquisition software.

\section{References}

Ade, H., X. Zhang, S. Cameron, C. Costello, J. Kirz, and S. Williams (1992), Chemical contrast in X-ray microscopy and spatially resolved XANES spectroscopy of organic specimens, Science, 258, 972-975.

Ankudinov, A. L., B. Ravel, J. J. Rehr, and S. D. Conradson (1998), Real-space multiple-scattering calculation and interpretation of x-ray-absorption near-edge structure, Phys. Rev. $B, 58,7565-7576$.

Berry, A. J., H. St. C. O’Neill, K. D. Jayasuriya, S. J. Campbell, and G. J. Foran (2003), XANES calibrations for the oxidation state of iron in a silicate glass, Am. Mineral., 88,967-977.

Buschert, R., M. D. Giardina, A. Merlini, A. Balerna, and S. Mobilio (1988), Laboratory EXAFS in a dispersive mode, J. Appl. Crystallogr., 21, 79-85.

De Andrade, V., O. Vidal, E. Lewin, and P. O'Brien (2006), Quantification of electron microprobe compositional maps of rock thin sections: An optimized method and examples, J. Metamorph. Geol., 24, 655-668.

Dyar, M. D., M. E. Gunter, J. S. Delaney, A. Lanzirotti, and S. R. Sutton (2002a), Use of spindle stage for orientation of single crystals for microXAS: Isotropy and anisotropy in Fe-XANES spectra, Am. Mineral., 87, 1500-1504.

Dyar, M. D., E. W. Lowe, C. V. Guidotti, and J. S. Delaney $(2002 \mathrm{~b}), \mathrm{Fe}^{3+}$ and $\mathrm{Fe}^{2+}$ partitioning among silicates in metapelites: A synchrotron micro-XANES study, Am. Mineral., 87, 514-522.

Farges, F., G. E. Brown Jr., P.-E. Petit, and M. Muñoz (2001), Transition elements in water-bearing silicate glasses/melts. Part I. A high-resolution and anharmonic analysis of $\mathrm{Ni}$ coordination environments in crystals, glasses, and melts, Geochim. Cosmochim. Acta, 65, 1665-1678.

Lytle, F. W., D. E. Sayers, and E. A. Stern (1975), Extended X-ray-absorption fine-structure technique. II. Experimental practice and selected results, Phys. Rev. B, 11, 4825-4835. 
Manceau, A., D. Bonnin, P. Kaiser, and C. Frétigny (1988), Polarized EXAFS of biotite and chlorite, Phys. Chem. Miner., 16, 180-185.

Matsushita, T., and R. P. Phizackerley (1981), A fast X-ray absorption spectrometer for use with synchrotron radiation, Jpn. J. Appl. Phys., 20, 2223-2228.

Muñoz, M., P. Argoul, and F. Farges (2003), Continuous Cauchy wavelet transform analyses of EXAFS spectra: A qualitative approach, Am. Mineral., 88, 694-700.

Muñoz, M., H. Bureau, V. Malavergne, B. Ménez, M. Wilke, C. Schmidt, A. Simionovici, A. Somogyi, and F. Farges (2005), In situ speciation of nickel in hydrous melts exposed to extreme conditions, Phys. Scr. T, 115, 921-922.

Pascarelli, S., T. Neisius, and S. De Panfilis (1999), TurboXAS: Dispersive XAS using sequential acquisition, J. Synchrotron Radiat., 6, 1044-1050.

Pascarelli, S., O. Mathon, M. Muñoz, T. Mairs, and J. Susini (2006), Energy-dispersive absorption spectroscopy for hardX-ray micro-XAS applications, J. Synchrotron Radiat., 13, $351-358$.

Pfalzer, P., J.-P. Urbach, M. Klemm, and S. Horn (1999), Elimination of self-absorption in fluorescence hard-x-ray absorption spectra, Phys. Rev. B, 60, 9335-9339.

Sayers, D. E., F. W. Lytle, and E. A. Stern (1970), Point scattering theory of X-ray K-absorption fine structure, $A d v$. X-ray Anal., 13, 248-271.
Shulman, R. G., Y. Yafet, P. Eisenberger, and W. E. Blumberg (1976), Observation and interpretation of x-ray absorption edges of iron compounds and proteins, Proc. Natl. Acad. Sci. U. S. A., 73, 1384-1388.

Sutton, S. R., S. Bajt, J. Delaney, D. Schuze, and T. Tokunaga (1995), Synchrotron x-ray fluorescence microprobe: Quantification and mapping of mixed valence state samples using micro-XANES, Rev. Sci. Instrum., 66, 1464-1467.

Teo, B. K. (1986), EXAFS: Basic Principles and Data Analysis, 9th ed., Inorg. Chem. Concepts, vol. 9, 349 pp., Springer, New York.

Vidal, O., T. Parra, and P. Vieillard (2005), Thermodynamic properties of the Tschermak solid solution in Fe-chlorite: Application to natural examples and possible role of oxidation, Am. Mineral., 90, 347-358.

Vidal, O., V. De Andrade, E. Lewin, M. Muñoz, T. Parra, and S. Pascarelli (2006), P-T-deformation- $\mathrm{Fe}^{3+} / \mathrm{Fe}^{2+}$ map at the thin section scale using Chl-Mica local equilibria and comparison with XANES mapping: Application to a garnetbearing metapelite from Sambagawa (Japan), J. Metamorph. Geolo., 24, 669-683.

Wilke, M., F. Farges, P.-E. Petit, G. E. Brown Jr., and F. Martin (2001), Oxidation state and coordination of $\mathrm{Fe}$ in minerals: An Fe K-XANES spectroscopic study, Am. Mineral., 86, $714-730$ 